\title{
Nonlinear Patterns in Chemical Networks as Symmetries of Transformation Groups
}

\author{
H.-G. Busse and B. Havsteen \\ Biochemisches Institut im Fachbereich Medizin der Universität Kiel \\ Z. Naturforsch. 32a, 1457-1464 [1977]; received July 9, 1977
}

\begin{abstract}
The origin of a stable spatial pattern in chemical reaction systems has been traced back to symmetries characterized by one-parameter transformation groups. The chemical reactiondiffusion system of Dreitlein and Smoes served as a model for the mathematical approach. The procedure is based on the conversion of sets of partial differential equations to equivalent systems of first order in more parameters. The latter generally admit one-parameter groups, which are related to singularities observed as pattern. It is also shown how models may be constructed from Lie-algebras using the constraints of integrability.
\end{abstract}

\section{Introduction}

Pattern formation in nonlinear dynamic systems is a difficult subject to study. Not only are the mathematical descriptions cumbersome, if possible at all, but also the corresponding experiments sometimes display unexpected effects. In physics, a classical example of a pattern formation of this type is the array of hexagonal cells formed in the Benard [1] experiment. The hexagonal cell pattern in a liquid is maintained by a temperature gradient perpendicular to the surface. The hydrodynamic state of the circulating flow of the liquid within the cells may be described by a set of nonlinear partial differential equations. The same set also applies to pattern phenomena like turbulence in vortices [2] and to nonlinear waves. Similar difficulties are encountered in weather forecasting. These are only a few examples of patterns arising from nonlinear processes, which are found throughout the cosmos from its micro to its macro scale.

In the present paper, pattern formation in chemical reaction systems is treated. The basic procedure used is an extension of the methods developed in a previous article [3]. Firstly, the reaction-diffusion equation proposed by Dreitlein and Smoes [4] is considered as an example. The solutions of the system of partial differential equations are considered as a continuous multi-parameter group, the elements of which are represented by Lie series. This concept allows the recast of the set of partial differential equations in a system of nonlinear differential equations of first order in

Requests for reprints should be sent to Prof. B. Havsteen, Biochemisches Institut im Fachbereich Medizin der Universität Kiel, Olshausenstr. 40-60, D-2300 Kiel. more than one parameter. Subsequently, oneparameter transformation groups of the system can be found, which leave the latter system invariant. These groups are related to singular solutions, as already pointed out in the previous paper [3]. The relation of the spatio-temporal pattern to singular solutions is derived from the chemical model. The generalization of these results demonstrates the importance of Lie-algebras and the integrability conditions in this endeavour.

Mathematical details of transformation groups [5] and vector spaces [6] are presented elsewhere and will not be reviewed here. Finally, the notation of vector spaces is used to summarize the conditions derived for singularities in equations of forms.

\section{The Reaction-Diffusion Model}

The kinetics and spatio-temporal distribution of a two-variable model has been studied by Dreitlein and Smoes [4]. The nonlinear partial differential equations of the system are given in their dimensionless form by [7]:

$$
\begin{aligned}
\frac{\partial}{\partial t} a_{1} & -D_{1} \frac{\partial^{2}}{\partial x^{2}} a_{1}=\left(E-a_{1}{ }^{2}-a_{2}{ }^{2}\right) a_{1} \\
& +S a_{2}-2 R a_{1}=F_{1}, \\
\frac{\partial}{\partial t} a_{2} & -D_{2} \frac{\partial^{2}}{\partial x^{2}} a_{2}=\left(E-a_{1}{ }^{2}-a_{2}{ }^{2}\right) a_{2} \\
& -S a_{1}+2 R a_{2}=F_{2},
\end{aligned}
$$

where the dependent variables $a_{1}, a_{2}$ are species quantities (component densities). The parameters $D_{1}, D_{2}, E, S$, and $R$ are real constants and $t, x$ are independent variables corresponding to time and space, respectively. 
In the following, the right hand side of Eqs. (1) is abbreviated as $F_{1}$ and $F_{2}$ respectively.

Equations (1) are equivalent to the following system of differential equations of first order in two independent variables $(t, x)$ :

$$
\begin{aligned}
& \frac{\partial}{\partial t} a_{1}=a_{3}, \quad \frac{\partial}{\partial t} a_{2}=a_{4}, \\
& \frac{\partial}{\partial x} a_{1}=a_{5}, \quad \frac{\partial}{\partial x} a_{2}=a_{6}, \\
& \frac{\partial}{\partial x} a_{5}=\frac{1}{D_{1}}\left(a_{3}-F_{1}\right), \\
& \frac{\partial}{\partial x} a_{6}=\frac{1}{D_{2}}\left(a_{4}-F_{2}\right),
\end{aligned}
$$

where $a_{i}(i=3,4,5,6)$ are defined by the upper four equations.

Equations (2a) may be complemented by the equations:

$$
\begin{aligned}
& \frac{\partial}{\partial t} a_{3}=g_{1}, \quad \frac{\partial}{\partial t} a_{4}=g_{2}, \quad \frac{\partial}{\partial t} a_{5}=g_{3}, \\
& \frac{\partial}{\partial t} a_{6}=g_{4}, \quad \frac{\partial}{\partial x} a_{3}=g_{5}, \quad \frac{\partial}{\partial x} a_{4}=g_{6},
\end{aligned}
$$

where the functions $g_{i}(i=1, \ldots, 6)$ satisfy the integrability conditions

$$
\begin{aligned}
& \partial^{2} a_{1} / \partial x \partial t=\partial^{2} a_{1} / \partial t \partial x ; \\
& \partial^{2} a_{2} / \partial x \partial t=\partial^{2} a_{2} / \partial t \partial x \quad \text { etc. }
\end{aligned}
$$

It follows that:

$$
\begin{aligned}
g_{5} & =g_{3} ; \quad g_{6}=g_{4} ; \quad \frac{\partial}{\partial x} g_{1}=\frac{\partial}{\partial t} g_{3} ; \\
\frac{\partial}{\partial x} g_{2} & =\frac{\partial}{\partial t} g_{4}, \\
\frac{\partial}{\partial x} g_{3} & =\frac{\partial}{\partial t}\left\{\frac{1}{D_{1}}\left(a_{3}-F_{1}\right)\right\}=\frac{1}{D_{1}}\left\{-\frac{\partial F_{1}}{\partial t}+g_{1}\right\}, \\
\frac{\partial}{\partial x} g_{4} & =\frac{\partial}{\partial t}\left\{\frac{1}{D_{2}}\left(a_{4}-F_{2}\right)\right\}=\frac{1}{D_{2}}\left\{-\frac{\partial F_{2}}{\partial t}+g_{2}\right\} .
\end{aligned}
$$

Thus, we have four equations for the four unknown functions: $g_{i}(i=1,2,3,4)$.

Solutions of the system (2) of differential equations may be interpreted as transformations of initial points $a^{0}=\left\{a_{1}^{0}, a_{2}^{0}, a_{3}^{0}, a_{4}^{0}, a_{5}{ }^{0}, a_{6}{ }^{0}\right\}$ into final points $a=\left\{a_{1}, a_{2}, a_{3}, a_{4}, a_{5}, a_{6}\right\}$ belonging to a parameter set $(x, t)$. Such a transformation can be carried out by two infinitesimal generators, $A_{x}$, $A_{t}$, associated to $x$ and $t$, respectively. The finite transformation is then given by a multi-dimensional Lie-series :

$$
a_{i}\left(x, t, a^{0}\right)=\left.\sum_{v=0}^{\infty} \frac{1}{v !}\left(t A_{t}+x A_{x}\right)^{v} a_{i}\right|_{a=a_{0}},
$$

where $a_{i}$ is a variable element of $a$.

The system of differential equations of first order in the independent variables $(x, t)$ generated by the Lie series (4) is [8]:

$$
\frac{\partial a_{i}}{\partial t}=\xi_{i} ; \quad \frac{\partial a_{i}}{\partial x}=\eta_{i},
$$

where the functions $\xi_{i}, \eta_{i}$ depend on $a_{i}$.

The associated infinitesimal generators are:

$$
A_{t}=\sum_{i=1}^{6} \xi_{i} \frac{\partial}{\partial a_{i}} ; \quad A_{x}=\sum_{i=1}^{6} \eta_{i} \frac{\partial}{\partial a_{i}} .
$$

Comparison of Eq. (5a) with Eqs. (2) gives the generators $(5 \mathrm{~b})$ by combining the equations with $\partial / \partial t$ and $\partial / \partial x$, respectively:

$$
\begin{aligned}
& A_{t}= a_{3} \frac{\partial}{\partial a_{1}}+a_{4} \frac{\partial}{\partial a_{2}}+g_{1} \frac{\partial}{\partial a_{3}}+g_{2} \frac{\partial}{\partial a_{4}} \\
&+g_{3} \frac{\partial}{\partial a_{5}}+g_{4} \frac{\partial}{\partial a_{6}}, \\
& A_{x}=a_{5} \frac{\partial}{\partial a_{1}}+a_{6} \frac{\partial}{\partial a_{2}}+g_{5} \frac{\partial}{\partial a_{3}}+g_{6} \frac{\partial}{\partial a_{4}} \\
&+\frac{1}{D_{1}}\left(a_{3}-F_{1}\right) \frac{\partial}{\partial a_{5}}+\frac{1}{D_{2}}\left(a_{4}-F_{2}\right) \frac{\partial}{\partial a_{6}} .
\end{aligned}
$$

The integrability condition for the system $(5 \mathrm{a}, \mathrm{b})$ requires that the commutator of the generators $A_{t}$ and $A_{x}$ satisfies [8]:

$$
\left[A_{t}, A_{x}\right]=0 .
$$

Equation (7) ensures that $x$ and $t$ define a surface on which one reaches the same point by proceeding first along $x$ and then along $t$ as when one follows first $t$ and then $x$. An explicit calculation of Eq. (7) leads to an equivalent set of Eqs. (3), which are:

$$
\begin{aligned}
& g_{5}=g_{3}, \quad g_{6}=g_{4}, \\
& A_{t} g_{3}=A_{x} g_{1}, \quad A_{t} g_{4}=A_{x} g_{2}, \\
& A_{x} g_{3}=-\frac{1}{D_{1}}\left\{a_{3} \frac{\partial F_{1}}{\partial a_{1}}+a_{4} \frac{\partial F_{1}}{\partial a_{2}}-g_{1}\right\}, \\
& A_{x} g_{4}=-\frac{1}{D_{2}}\left\{a_{3} \frac{\partial F_{2}}{\partial a_{1}}+a_{4} \frac{\partial F_{2}}{\partial a_{2}}-g_{2}\right\} .
\end{aligned}
$$


If the Eqs. (8c,d) are solved with respect to the functions $g_{1}$ and $g_{2}$, one obtains the following Eqs. (8b) in $g_{3}$ and $g_{4}$ :

$$
\begin{aligned}
a_{3} \frac{\partial g_{3}}{\partial a_{1}}+a_{4} \frac{\partial g_{3}}{\partial a_{2}}+\left(F_{1}^{\prime}+D_{1} A_{x} g_{3}\right) \frac{\partial g_{3}}{\partial a_{3}}+\left(F_{2}{ }^{\prime}+D_{2} A_{x} g_{4}\right) \frac{\partial g_{3}}{\partial a_{4}}+g_{3} & \frac{\partial g_{3}}{\partial a_{5}}+g_{4} \frac{\partial g_{3}}{\partial a_{6}} \\
& =A_{x} F_{1}{ }^{\prime}+D_{1} A_{x}^{2} g_{3}, \\
a_{3} \frac{\partial g_{4}}{\partial a_{1}}+a_{4} \frac{\partial g_{4}}{\partial a_{2}}+\left(F_{1}^{\prime}+D_{1} A_{x} g_{3}\right) \frac{\partial g_{4}}{\partial a_{3}}+\left(F_{2}{ }^{\prime}+D_{2} A_{x} g_{4}\right) \frac{\partial g_{4}}{\partial a_{4}}+g_{3} \frac{\partial g_{4}}{\partial a_{5}}+g_{4} \frac{\partial g_{4}}{\partial a_{6}} & =A_{x} F_{2}{ }^{\prime}+D_{2} A_{x}^{2} g_{4},
\end{aligned}
$$

where

$$
F_{1}^{\prime}=a_{3} \frac{\partial F_{1}}{\partial a_{1}}+a_{4} \frac{\partial F_{1}}{\partial a_{2}} \quad \text { and } \quad F_{2}{ }^{\prime}=a_{3} \frac{\partial F_{2}}{\partial a_{1}}+a_{4} \frac{\partial F_{2}}{\partial a_{2}} .
$$

The Eqs. (9) are nonlinear in the derivatives and hence are still more complicated than the Equations (1).

When the Eqs. (9) are solved, it is possible to calculate solutions to the Eqs. (1) using the Eqs. (4) and (5). If the Eqs. (9) do not have a solution, then this method is not possible, since Eq. (7) in that case is an invalid condition.

\section{One-Parameter Group of Transformations of Solutions}

Assuming that the functions $g_{1}, \ldots, g_{6}$ and the generators $A_{t}$ and $A_{x}$ have been determined, one may search for groups transforming solutions of Eqs. (2) into their like. The generator $U$ of such a transformation group requires:

$$
\begin{aligned}
& {\left[U, A_{t}\right]=\lambda_{1} A_{t}+\lambda_{2} A_{x} \text { and }} \\
& {\left[U, A_{x}\right]=\lambda_{3} A_{t}+\lambda_{4} A_{x},}
\end{aligned}
$$

where $\lambda_{i}(i=1, \ldots, 4)$ depends on $a_{k}(k=1,2, \ldots, 6)$. From a previous treatment $[3]$ of the model we know that:

$$
U^{r}=a_{2} \frac{\partial}{\partial a_{1}}-a_{1} \frac{\partial}{\partial a_{2}}
$$

is a generator for space independent solutions of Eqs. (1) with $R=0$. Moreover, it is a generator in

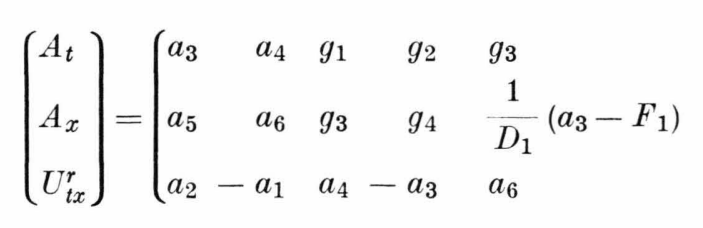

the space-dependent case with $R=0$ and $D_{1}=$ $D_{2}=D$. To verify this, $U^{r}$ has to be once-extended [9] with respect to time and space to act in the space of $a_{i}$.

$$
\begin{aligned}
U_{t x}^{r}= & a_{2} \frac{\partial}{\partial a_{1}}-a_{1} \frac{\partial}{\partial a_{2}}+a_{4} \frac{\partial}{\partial a_{3}}-a_{3} \frac{\partial}{\partial a_{4}} \\
& +a_{6} \frac{\partial}{\partial a_{5}}-a_{5} \frac{\partial}{\partial a_{6}}
\end{aligned}
$$

The generator:

$$
\begin{aligned}
U_{t x x}^{r}= & a_{2} \frac{\partial}{\partial a_{1}}-a_{1} \frac{\partial}{\partial a_{2}}+a_{4} \frac{\partial}{\partial a_{3}}-a_{3} \frac{\partial}{\partial a_{4}} \\
& +a_{7} \frac{\partial}{\partial a_{8}}-a_{8} \frac{\partial}{\partial a_{7}}
\end{aligned}
$$

which is once-extended with respect to time and twice-extended with respect to space leaves the Eqs. (1) invariant with respect to their form. The parameters $a_{7}=\partial^{2} c_{1} / \partial x^{2}$ and $a_{8}=\partial^{2} c_{2} / \partial x^{2}$ have to be substituted in Eqs. (1) to verify this. It may be mentioned that also the generators $\partial / \partial t$ and $\partial / \partial x$ leave Eqs. (1) invariant.

\section{Degeneracy of Infinitesimal Generators}

The generators $A_{t}, A_{x}$, and $U_{t x}^{r}$ can be cast into matrix form:

$$
\left.\begin{array}{l}
g_{4} \\
-a_{5} \\
\left.-a_{4}-F_{2}\right) \\
\partial / \partial a_{4} \\
\partial / \partial a_{5} \\
\partial / \partial a_{6}
\end{array}\right) .
$$


If the generators become linear dependent, then the system of the three generators is degenerated. Firstly, we investigate the case where $A_{t}=\lambda A_{x}$. It follows that all $2 \times 2$ determinants of the first two rows in the matrix of the right-hand side of Eq. (12) vanish. Hence, using:

$$
\lambda=w / k
$$

we obtain:

$$
\begin{aligned}
& a_{3}=\frac{w}{k} a_{5} ; \quad a_{4}=\frac{w}{k} a_{6}, \\
& g_{1}=\frac{w}{k} g_{3} ; \quad g_{2}=\frac{w}{k} g_{4}, \\
& g_{3}=\frac{w}{k} \frac{1}{D_{1}}\left(a_{3}-F_{1}\right), \\
& g_{4}=\frac{w}{k} \frac{1}{D_{2}}\left(a_{4}-F_{2}\right)
\end{aligned}
$$

and the degenerate Equations (2):

$$
\begin{aligned}
& \frac{\partial a_{1}}{\partial t}=a_{3}=\frac{w}{k} a_{5}=\frac{\partial a_{1}}{\partial x} \frac{w}{k}, \\
& \frac{\partial a_{2}}{\partial t}=a_{4}=\frac{w}{k} a_{6}=\frac{\partial a_{2}}{\partial x} \frac{w}{k}, \\
& \frac{\partial a_{3}}{\partial t}=\frac{w^{2}}{k^{2}} \frac{1}{D_{1}}\left(a_{3}-F_{1}\right)=\frac{\partial a_{3}}{\partial x} \frac{w}{k}, \\
& \frac{\partial a_{4}}{\partial t}=\frac{w^{2}}{k^{2}} \frac{1}{D_{2}}\left(a_{4}-F_{2}\right)=\frac{\partial a_{4}}{\partial x} \frac{w}{k}, \\
& \frac{\partial a_{5}}{\partial t}=\frac{w}{k} \frac{1}{D_{1}}\left(a_{3}-F_{1}\right)=\frac{\partial a_{5}}{\partial x} \frac{w}{k}, \\
& \frac{\partial a_{6}}{\partial t}=\frac{w}{k} \frac{1}{D_{2}}\left(a_{4}-F_{2}\right)=\frac{\partial a_{6}}{\partial x} \frac{w}{k} .
\end{aligned}
$$

The condition $\partial x / \partial t=w / k$ yields the similarity variable:

$$
z=w t-k x .
$$

Because of the dependence of $a_{5}$ and $a_{6}$ on $a_{3}$ and $a_{4}$, Eqs. (14) reduce to:

$$
\begin{array}{ll}
\frac{\partial a_{1}}{\partial z}=\frac{a_{3}}{w}, & \frac{\partial a_{3}}{\partial z}=\frac{w}{D_{1} k^{2}}\left(a_{3}-F_{1}\right), \\
\frac{\partial a_{2}}{\partial z}=\frac{a_{4}}{w}, & \frac{\partial a_{4}}{\partial z}=\frac{w}{D_{2} k^{2}}\left(a_{4}-F_{2}\right),
\end{array}
$$

where the similarity variable $z$ is used as a parameter. The generator:

$$
\begin{aligned}
A_{z}= & \frac{a_{3}}{w} \frac{\partial}{\partial a_{1}}+\frac{a_{4}}{w} \frac{\partial}{\partial a_{2}}+\frac{w}{D_{1} k^{2}}\left(a_{3}-F_{1}\right) \\
& \cdot \frac{\partial}{\partial a_{3}}+\frac{w}{D_{2} k^{2}}\left(a_{4}-F_{2}\right) \frac{\partial}{\partial a_{4}}
\end{aligned}
$$

belongs to the Equations (15). The group $U_{t x}^{r}$ reduces to

$$
U_{z}=a_{2} \frac{\partial}{\partial a_{1}}-a_{1} \frac{\partial}{\partial a_{2}}+a_{4} \frac{\partial}{\partial a_{3}}-a_{3} \frac{\partial}{\partial a_{4}} .
$$

$A_{z}$ and $U_{z}$ degenerate to $A_{z}=\lambda_{z} U_{z}$ if

$$
\begin{aligned}
w \lambda_{z} & =S, \quad a_{3}=S a_{2}, \\
a_{4} & =-S a_{1}, \quad E-D k^{2} \lambda_{z}{ }^{2}=a_{1}{ }^{1}+a_{2}{ }^{2}
\end{aligned}
$$

admitting under Eqs. (15) the solution:

$$
\begin{aligned}
& a_{1}=\left(E-D k^{2}\right) \cos z, \\
& a_{2}=-\left(E-D k^{2}\right) \sin z,
\end{aligned}
$$

where

$$
w=S \quad \text { and } \quad \lambda_{z}=1
$$

which represents a propagating wave whose amplitude is dependent on the wave number $k$.

Now the case is studied where

$$
U=\lambda_{t} A_{t}+\lambda_{x} A_{x} \quad \text { and } \quad A_{t} \neq \lambda A_{x} .
$$

Consequently, all determinants of the three-row matrix in Eqs. (12) are zero. Whereas in the former case the conditions (13) lead to equations which are independent of the unknown functions $g_{i}$, the conditions here contain these functions.

(a) $\lambda_{t} a_{3}+\lambda_{x} a_{5}+a_{2}=0$,

(b) $\lambda_{t} a_{4}+\lambda_{x} a_{6}-a_{1}=0$,

(c) $\lambda_{t} g_{1}+\lambda_{x} g_{3}+a_{4}=0$,

(d) $\lambda_{t} g_{2}+\lambda_{x} g_{4}-a_{3}=0$,

(e) $\lambda_{t} g_{3}+\frac{\lambda_{x}}{D}\left(a_{3}-F_{1}\right)+a_{6}=0$,

(f) $\lambda_{t} g_{4}+\frac{\lambda_{x}}{D}\left(a_{4}-F_{2}\right)-a_{5}=0$.

Equations (a) and (b) determine $\lambda_{t}$ and $\lambda_{x}$ and Eqs. (c) through (f) $g_{1}, g_{2}, g_{3}$ and $g_{4}$. Equations (18) have to be compatible with Equations (8). In general, no functions $g_{i}$ will satisfy both sets of equations. However, Eqs. (18) may be considered as boundary conditions to Eqs. (8), if they are regarded as sets of partial differential equations in the independent variables $x$ and $t$. In that case, hypersurfaces in the parameter space may exist 
on which both sets are valid. The similarity variable $z=w t-k x$ is an example of such a surface.

\section{Generalization}

We assume that the set of infinitesimal generators $A_{i}(i=1,2, \ldots, m)$ form a Jacobian complete system [10], i.e.

$$
\left[A_{i}, A_{j}\right]=0 \quad(i, j=1,2, \ldots, m),
$$

and have a common solution. Moreover, there exist $n-m$ independent common solutions to all $A_{i}$ where $n$ is the number of variables $a_{k}$ of the $A_{i}$. The differential equation systems of first order associated with the $A_{i}=\sum_{k} \eta_{i k} \frac{\partial}{\partial a_{k}}$ is : $\frac{\partial a_{k}}{\partial t_{i}}=\eta_{i k} \quad(j, k=1,2, \ldots, n ; i=1,2, \ldots, m)$,

where $\eta_{i k}$ depends on $a_{j}$. It satisfies the integrability conditions (see Eqs. (3)). Hence, the set of parameters $\left(t_{i}\right)$ constitutes a unique coordinate system to represent the quantities $a_{k}$. The solutions of Eqs. (20) can be written as Lie-series:

$$
\begin{aligned}
a_{k}\left(t_{i}\right) & =\left.\exp \sum_{i}\left(t_{i} A_{i}\right) a_{k}\right|_{a_{k}=a_{k}{ }^{0}} \\
& =\left.\sum_{\nu=0}^{\infty} \frac{1}{v !}\left(\sum_{i} t_{i} A_{i}\right) a_{k}\right|_{a_{k}=a_{k}{ }^{0}}
\end{aligned}
$$

where $a_{k}^{0}$ are the initial values of the $a_{k}$. Within the domain of $t_{i}$, where the series converges, the latter represent single-valued unique functions. Moreover, because of the group properties, the inverse function exists with respect to the $\left\{t_{i}\right\}$.

By successive elimination of the $a_{k}$ 's from the system (20), systems of partial differential equations with mixed and higher order derivatives are obtained. Conversely, it is possible from a system of partial differential equations to construct systems of first order differential equations of the kind given in Eqs. (20). If the compatibility condition applies to this system, the latter can be treated as previously suggested. Since any complete system [10] can be transformed into a Jacobian complete system, one may construct partial differential equation systems of first order, the solutions of which form more-parameter transformation groups starting with any complete system.

The number $p$ of compatibility equations obtained from a system of $m$ parameters $t_{i}$ in the $n$ variables $a_{k}$ is given by:

$$
p=\frac{m(m-1)}{2} \cdot n .
$$

Since for $m=3$ the number of constraint equations already is equal to the number of $\eta_{i k}$ (compare Eq. (20)), the compatiblity conditions strongly restrict the equation set (20) for $m>3$.

The infinitesimal generator $U$ of a group may be used to transform the generators of the Jacobian complete system $A_{i}$ by commutation into:

$$
A_{i} *=\left[U, A_{i}\right] \text {. }
$$

Let $\lambda_{i j}$ be functions of the variables $a_{k}$ and

$$
A_{i} *=\sum_{j} \lambda_{i j} A_{j} \quad(i, j=1,2, \ldots, m),
$$

then the new generators $A_{i} *$ form a complete system having the same set of common solutions as $A_{j}$. Therefore, the group generated by $U$ transforms the set of solutions into themselves, assuming:

$$
\left[U, A_{i}\right]=\sum_{j} \lambda_{i j} A_{j} \quad(i, j=1,2, \ldots, m) .
$$

The transformation from $A$ to $A^{*}$ does not need to preserve the parameterization, since it uniquely maps solutions into other solutions, but not necessarily the parameter space into itself. The latter would require that the set of $\left\{A_{i} *\right\}$ also forms a Jacobian complete system. More than one $U$ may have this property (22). The number $l$ of independent $U$ 's has to be less than $n-m+1$, since otherwise the excessive $U$ 's would be dependent on the other $U$ 's through:

$$
U=\sum_{j=1}^{m} \lambda_{j} A_{j}+\sum_{s=1}^{n-m} \mu_{s} U_{s}
$$

(where $\lambda_{j}, \mu_{s}$ are functions of $a_{k}$ and $\mu_{s}$ common solutions to all $A_{i}$ ).

The main property of the considered set of solutions is the uniqueness inherent to its group character. Consequently, given any initial value, there is only one path connecting neighbouring values (in this case curves or surfaces cannot intersect). The uniqueness is abolished, if the generators of the group degenerate by becoming mutually dependent, e.g. :

$$
U=\sum_{j=1}^{m} \lambda_{j} A_{j}
$$

The set of values for which the uniqueness does not apply can be considered as singular. This set may 
be regarded as the boundary of a domain in which uniqueness is valid.

A differential equation system of first order:

$$
\frac{\partial}{\partial \tau_{s}} a_{k}=u_{s k} \quad\left(\tau_{s} \text { are parameters }\right)
$$

belongs to any independent

$$
U_{s}=\sum_{s} u_{s k} \frac{\partial}{\partial a_{k}} \quad(\text { see }(20)) .
$$

Eqs. (20) and (24) form a differential equation system in $m+1 \geqq n$ parameters $\left(t_{i}, \tau_{s}\right)$, which does not necessarily satisfy the compatibility conditions.

So far, uniqueness in the description has been the major topic. Another property of the physical laws is the independence of the coordinate system. In the following, we restate the former quality in the vector space notation [11] to stress its invariance with respect to coordinate transformations. In this notation, an infinitesimal generator is a vector field which assigns a vector to each point in space. Here, the vectors $a=\left(a_{1}, a_{2} \ldots a_{n}\right)$ with $\left(a \in M_{a}\right)$ constitute a manifold $M_{a}$ (the space) to which the vectors $A_{i} \in T_{a}$ are assigned. $T_{a}$ is the vector space of tangents at $a \in M_{a}$ whose basis is given by

$$
\left\{\frac{\partial}{\partial a_{k}}(k=1,2, \ldots, n)\right\} \text {. }
$$

The $\eta_{i k}$ (see Eq. (20)) is the vector component in the space of tangents. Multilinear maps, which map elements of a vector space onto the corresponding field, are called tensors. Alternating tensors form subspaces of tensor spaces, which are distinguished by their high symmetry. The alternating tensors of the tangent space are called forms. The basis of the vector space of one-forms is $\left\{\mathrm{d} a_{k}\right\}$. The basis of $\mathrm{h}$-forms can be expressed by the wedge product of the basis of one-forms, e.g. a 3 -form has the basis:

$$
\begin{aligned}
&\left\{\mathrm{d} a_{k} \wedge \mathrm{d} a_{i}\right. \wedge \mathrm{d} a_{j} \\
&(k>i>j ; k, i, j=1,2, \ldots, n)\} .
\end{aligned}
$$

A particular form is the $n$-form (the volume element) $w$ :

$$
w=w\left(a_{k}\right) \mathrm{d} a_{1} \wedge \mathrm{d} a_{2} \wedge \cdots \wedge \mathrm{d} a_{n} .
$$

The following three operations are defined in the vector space of forms: $L_{x} \alpha, \mathrm{d} \alpha$, and $\left.X\right\lrcorner \alpha$, where $L_{x}$ is the Lie derivative of the form $\alpha$ with respect to the vector field $X$ of the corresponding tangent space. The symbol $d$ is the exterior derivative of the form $\alpha$ and $X\lrcorner \alpha$ is the contraction of $\alpha$ with $X$.

Note that contraction converts a $h$-form into a $(h-1)$-form whereas an exterior derivation changes an $h$-form into an $(h+1)$-form.

Let $w\left(a_{k}\right)=1$, then $w$ is the unit volume element. By contraction of $w$ with vector fields, one obtains the $(n-1)$-forms :

$$
\begin{aligned}
\alpha_{i} & \left.=A_{i}\right\lrcorner w \quad(i=1,2, \ldots, m), \\
\mu & =U\lrcorner w .
\end{aligned}
$$

The condition $\left[U, A_{i}\right]=\lambda_{i j} A_{j}$ can now be rewritten as:

$$
L_{U} \alpha_{i}=\lambda_{i j^{\prime}} \alpha_{j} \quad(i, j=1,2, \ldots, m) .
$$

Thus, in coordinate-free form for

Eqs. (12): $L_{A_{i}} \alpha_{j}=0$,

Eqs. (22): $\quad L_{U} \alpha_{i}=\sum \lambda_{i j}{ }^{\prime} \alpha_{j}$,

Eqs. (23): $\quad U\lrcorner \beta=0$,

where $i, j=1,2, \ldots, m$ and

$$
\begin{aligned}
& \left.\alpha_{j}=A_{j}\right\lrcorner w \quad \text { and } \\
& \left.\left.\left.\left.\beta=A_{1}\right\lrcorner\left(A_{2}\right\lrcorner \cdots\right\lrcorner\left(A_{m}\right\lrcorner w\right) \cdots\right)
\end{aligned}
$$

are obtained.

The parameters $\left(t_{i}, \tau_{s}\right)$ can also form a manifold with the associated tangent space $T_{\tau}$. The basis of $T_{\tau}$ is given by

$\left\{\frac{\partial}{\partial t_{i}}, \quad \frac{\partial}{\partial \tau_{s}}(i=1,2, \ldots, m ; s=1,2, \ldots, l)\right\}$.

The dual space has the basis $\left\{\mathrm{d} t_{i}, \mathrm{~d} \tau_{s}\right\}$.

The dimension of the combined tangent spaces is maximally equal to $2 n$. The description of the physical processes may also be made in this combined space [12].

\section{Canonical Form of Groups and Coordinate Transformations}

Groups of transformations with more than one continuous parameter are produced by a generator $U$, which admits the representation:

$$
U=e_{1} U_{1}+e_{2} U_{2}+\cdots+e_{r} U_{r},
$$

where $e_{i}$ is a real constant and the $U_{i}(i=1,2, \ldots, r)$ forms a system of independent generators. The parameter $r$ is the number of continuous variables. 
Multi-parameter groups may be classified according to distinct relations, established by the Grassman (or Lie) algebra [13] of commutators and their independent generators. The two-parameter $(t, x)$ example admits the classification of two-parameter groups. There are four classes [10] (I) - (IV), satisfying the following conditions:
(I) $\left[U_{1}, U_{2}\right]=0, \quad U_{2} \neq \varrho U_{1}$,
(II) $\left[U_{1}, U_{2}\right]=0, \quad U_{2}=\varrho U_{1}$,
(III) $\left[U_{1}, U_{2}\right]=U_{1}, \quad U_{2} \neq \varrho U_{1}$,
(IV) $\left[U_{1}, U_{2}\right]=U_{1}, \quad U_{2}=\varrho U_{1}$,

where $\varrho$ is a function of the variables of the $U$ 's.

The generators of the classes (I) and (II) of Eqs. (26) are given in the canonical variables $u_{1}$ and $u_{2}$ by:

$$
\begin{aligned}
\text { (I) } U_{1}=\partial / \partial u_{1}, & U_{2}=\partial / \partial u_{2}, \\
\text { (II) } \quad U_{1}=\partial / \partial u_{2}, & U_{2}=u_{1} \partial / \partial u_{2} .
\end{aligned}
$$

The commutator vanishes for both classes, i.e. they form a Jacobian complete system.

Changing to new variables $a_{i}$ by the transformation:

$$
a_{i}=a_{i}\left(u_{1}, u_{2}\right) \quad(i=1,2, \ldots, n)
$$

leads to the generators $U_{1} *, U_{2} *$ according to [10]:

$$
\begin{aligned}
& U_{1} *=\sum_{i=1}^{n}\left(U_{1} a_{i}\right) \frac{\partial}{\partial a_{i}} ; \\
& U_{2} *=\sum_{i=1}^{n}\left(U_{2} a_{i}\right) \frac{\partial}{\partial a_{i}} .
\end{aligned}
$$

Evidently, the commutator of the generators $U_{1}$ * and $U_{2} *$ vanishes, i.e.

$$
\left[U_{1}^{*}, U_{2}^{*}\right]=0
$$

since it is preserved under coordinate transformations. Hence, $U_{1} *$ and $U_{2} *$ also form a Jacobian complete system. In the same manner, Jacobian complete systems may be constructed from canonical forms of multi-parameter groups with more than two parameters. In turn, these Jacobian complete systems may be used as described in the previous section.

[1] S. Chandrasekhar, Hydrodynamic and Hydromagnetic Stability, Clarendon Press, Oxford 1961.

[2] L. D. Landau and E. M. Lifshitz, Fluid Mechanics, Pergamon Press, London 1959.

\section{Discussion}

The criteria developed for limit cycles in a previous paper [3] may, as the example shows, be extended to systems involving spatial coordinates. A one-parameter group may still be found which transforms solutions of the defining differential equations into their like. The process of transformation becomes singular if the solution is an invariant function of the group. Invariant functions are transformed into themselves by the transformations of the group. Thus, solutions which are symmetrical with respect to the one-parameter group reveal the special properties of limit cycles. They are independent of the parameter that transforms solutions into new solutions. Since the parameters, which fix a solution, are initial and boundary data, the symmetrical solution is independent of some of these data.

In a multi-dimensional case, the condition of symmetry severely restricts the solutions. Therefore, a solution requires a prescribed boundary in the parameter space $(x, t)$. This phenomenon may be interpreted as structure generation in the space of parameter variables. Some processes of morphogeneses in biology [14] might be explained by such phenomenon. In this context, it should be mentioned that the compatibility conditions also greatly restrict the set of models allowed. Moreover, they strongly constrain the space of parameter variables $(x, t)$ for dimensions greater than three. Since compatibility is closely related to Lie-algebras, the models may be arranged according to the classification of the associated Lie-algebra.

The preceding sections only treat dynamical aspects of chemical or similar models. The latter are often related to the thermodynamics of irreversible processes. The structura! and energetic views of thermodynamics would supply additional characteristics. Particularly the boundary operation would implicate new useful relations.

Finally, we emphasize that the description outlined is not restricted to chemical networks, but may also be applied to systems of other species and quantities. Systems generating discrete parameter groups are probably of considerable interest for analysis, e.g. of interacting biological systems.

[3] H. G. Busse and B. Havsteen, Z. Naturforsch. 32a, 40 (1977).

[4] J. Dreitlein and M. L. Smoes, J. Theor. Biol. 46, 559 (1974). 
[5] L. P. Eisenhart, Continuous Groups of Transformations, Dover, New York 1961.

[6] M. Spivak, Calculus on Manifolds, Benjamin, New York 1965.

[7] G. Rosen J. Theor. Biol. 54, 391 (1975).

[8] G. Wanner, Theorie der Lie-Reihen, In: D. Laugwitz, Utberblicke Mathematik, Bd. 1, BI Verlag, Mannheim 1968.

[9] G. W. Bluman and J. D. Cole, Similarity Methods for Differential Equations, Appl. Math. Sciences Vol. 13, Springer-Verlag, Berlin 1974.
[10] A. Cohen, An Introduction to the Lie Theory of Oneparameter Groups, Heath \& Co., Boston 1911.

[11] R. M. Kiehn, Int. J. Engng. Sci. 13, 941 (1975).

[12] B. K. Harrison and F. B. Estabrook, J. Math. Phys. 12, 653 (1971).

[13] R. Hermann, Differential Geometry and the Calculus of Variations, Academic Press, New York 1968.

[14] H. G. Busse and B. Havsteen, Recast of Large Scale Networks for Reduction, submitted to J. Theor. Biol. 